\title{
Electromagnetic compatibility (EMC) evaluation of the SELENE spacecraft for the lunar radar sounder (LRS) observations
}

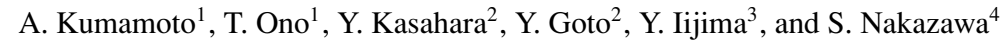 \\ ${ }^{1}$ Graduate School of Science, Tohoku University, 6-3, Aoba, Aramaki, Aoba, Sendai 980-8578, Japan \\ ${ }^{2}$ Graduate School of Natural Science and Technology, Kanazawa University, 2-40-20, Kakuma-machi, Kanazawa 920-1192, Japan \\ ${ }^{3}$ Institute of Space and Astronautical Science, Japan Aerospace Exploration Agency, 3-1-1, Yoshinodai, Sagamihara 229-8510, Japan \\ ${ }^{4}$ Tsukuba Space Center, Japan Aerospace Exploration Agency, 2-1-1, Sengen, Tsukuba 305-8505, Japan
}

(Received March 16, 2007; Revised July 12, 2007; Accepted August 3, 2007; Online published April 9, 2008)

\begin{abstract}
In order to achieve the lunar subsurface sounding and planetary radio wave observations by the Lunar Radar Sounder (LRS) onboard the SELENE spacecraft, strict electromagnetic compatibility (EMC) requirements were applied for all instruments and the whole system of the spacecraft. In order to detect the lunar subsurface echoes from a depth of $5 \mathrm{~km}$, the radiated emission (RE) limit was determined to be $-10 \mathrm{~dB} \mu \mathrm{V} / \mathrm{m}$ and the commonmode $(\mathrm{CM})$ current limit to be $20 \mathrm{~dB} \mu \mathrm{A}$. The EMC performance of the spacecraft was finally evaluated in the system EMC test held from Oct. 20 to Oct. 22, 2005. There is no broadband noise but some narrowband noises at a level above the CM-current limit in a frequency range from 4 to $6 \mathrm{MHz}$, in which radar soundings are operated. Based on the noise spectrum within 4-6 MHz, the noise level of FMCW radar sounder is estimated to be $14 \mathrm{~dB}$ lower than the CM-current limit. In the SELENE EMC test, the following new techniques were introduced: (1) systematic control and evaluation of CM-current noises were first performed to improve the spacecraft EMC performance; (2) onboard battery operation was utilized for reduction of ambient broadband noises during EMC measurements.
\end{abstract}

Key words: SELENE spacecraft, lunar radar sounder (LRS), subsurface radar sounding, electromagnetic compatibility (EMC), radiated emission (RE) noise, common-mode current noise.

\section{Introduction}

Global survey of the lunar subsurface structure by the lunar radar sounder (LRS) is one of the most challenging objectives of the SELENE mission (Ono and Oya, 2000; Ono et al., 2008). In order to detect weak lunar subsurface echoes and to perform advanced analyses (Kobayashi et al., 2002a, b; Kobayashi and Ono, 2006, 2007), the artificial noises originating from the SELENE spacecraft system itself should be suppressed adequately below the estimated subsurface echo level. Not only radar soundings of the lunar subsurface structure but also observations of planetary radio waves, such as Jovian hectometric (HOM) and decametric (DAM) emissions, and plasma waves around the moon are planned using the LRS high sensitive receiver.

The instrumentation of the LRS has been described in detail by Ono and Oya (2000) and Ono et al. (2008). The LRS consists of four antenna units (LRS-A1, A2, A3, and A4), four preamplifier units (LRS-P1, P2, P3, and P4), and the main electric circuit unit (LRS-E). An overview of the SELENE spacecraft is shown in Fig. 1. Antenna units and corresponding preamplifier units are set together in four brackets and installed on the inner side of the $+\mathrm{Z}$ panel, which is always oriented to the moon center. LRS-A1/P1 (X1 antenna) and LRS-A4/P4 (Y2 antenna) are near the -Y panel,

Copyright (c) The Society of Geomagnetism and Earth, Planetary and Space Sciences (SGEPSS); The Seismological Society of Japan; The Volcanological Society of Japan; The Geodetic Society of Japan; The Japanese Society for Planetary Sciences; TERRAPUB. while LRS-A2/P2 (Y1 antenna) and LRS-A3/P3 (X2 antenna) are near the $+Y$ panel. The antenna elements are fully retracted when the spacecraft is launched. After the spacecraft is injected into the lunar orbit, the antenna elements are extended up to a length of $15 \mathrm{~m}$ and used for carrying out lunar subsurface radar soundings in a frequency range of 4 to $6 \mathrm{MHz}$ and making observations of natural radio and plasma waves in a frequency range up to $30 \mathrm{MHz}$.

The artificial noises that affect the observations for scientific purposes are categorized into two groups: radiated emission (RE) noises and conducted emission (CE) noises. RE noises appear as an unfavorable electric field emitted from the components and cables onboard the spacecraft. CE noises take the form of an unfavorable current which disturbs other instruments via the interconnecting cables. Especially in the case of the spacecraft, common-mode (CM) currents passing through the spacecraft panels cause EMC problems. Most spacecraft BUS equipments and scientific instruments consist of separated components. For example, the lunar magnetic field and particle plasma (MAP) instrument consists of magnetometer, mast deployment equipment, four particle detectors, and the main electric circuit unit installed on the different spacecraft panels. The components are connected with cables, or several sets of HOT and RTN lines. When the HOT and RTN currents in the cables are balanced each other, there is no current and no potential difference between the spacecraft panels. In practice, however, the HOT and RTN currents are not balanced 


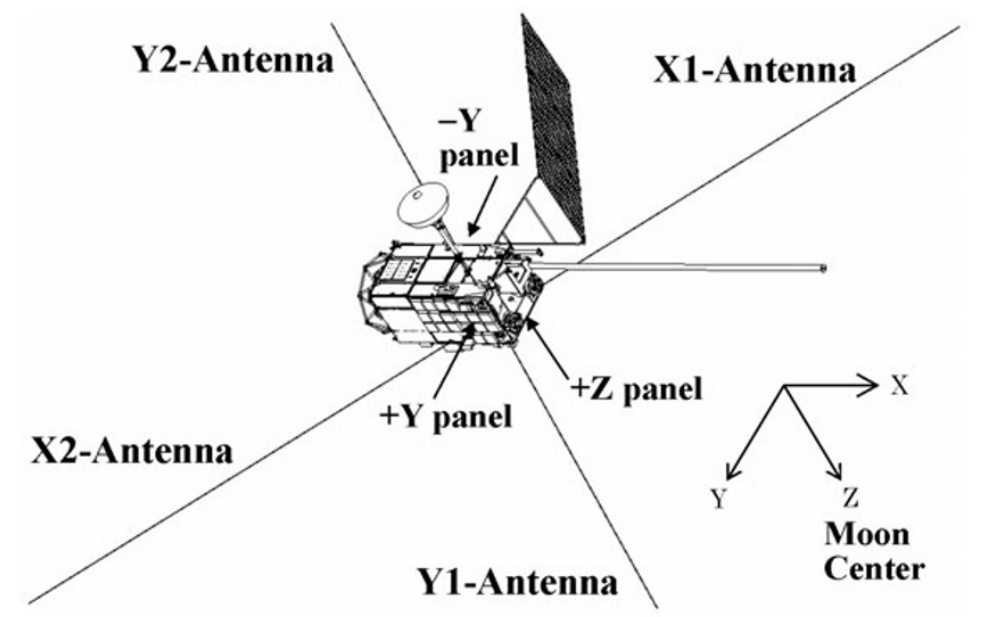

Fig. 1. Overview of the locations of the spacecraft panels and LRS antenna elements. The $\mathrm{Z}$ axis is directed toward the moon center; the $\mathrm{X}$ axis is aligned with the orbit.

and impedance between the spacecraft panels is not $0 \Omega$ in whole frequency range up to $30 \mathrm{MHz}$. CM currents appear in the spacecraft panels and produce the potential difference between the LRS preamplifier inputs. The CM currents in the spacecraft panels can be evaluated by making measurements of unbalanced currents in the interconnecting cables between the instrument components.

In order to suppress the artificial noises mentioned above, strict EMC requirements were applied for all components and the whole system of the SELENE spacecraft. The techniques for EMC control and EMC evaluation of the spacecraft with scientific payloads have been improved through the trials made for the Akebono (Kimura et al., 1990; Oya et al., 1990), Geotail (Tsutsui et al., 1992; Matsumoto et al., 1994), and Nozomi (Ono et al., 1998; Matsumoto et al., 1998) missions, and the methodology of the SELENE EMC control is based on them. It is especially based on the EMC investigation of the Nozomi power supply unit, where it was recognized that the EMC performance of the spacecraft is highly dependent on the CM-current noises in the interconnecting cables between instruments. The total EMC performance of the SELENE spacecraft was finally checked in the system EMC test held from October 20 to 22, 2005 in the anechoic chamber of the Tsukuba Space Center (TKSC), Japan Aerospace and Exploration Agency (JAXA). In this paper, the results of SELENE EMC control for the lunar subsurface radar sounding are presented in detail. The basis of SELENE EMC standard and methodology of EMC controls are described in Section 2. The results of the final system EMC test are shown in Section 3, and the discussion and conclusions are presented in Section 4.

\section{EMC Requirements for the SELENE Space- craft}

The galactic noise level is considered to be a criterion for detection of the lunar the subsurface echo by the LRS. Based on the feasibility study by Ono and Oya (2000), the intensity of subsurface echo from a depth of $5 \mathrm{~km}$ is estimated to be larger than $-98.6 \mathrm{dBm}$, which is almost equal to galactic nose level at $5 \mathrm{MHz}$, in the case that the loss tan- gent $\tan \delta=\operatorname{Im} \varepsilon / \operatorname{Re} \varepsilon$, the ratio of real and imaginary parts of permittivity is smaller than 0.006. Based on recent observations of galactic background radiations in HF ranges (Dulk et al., 2001; Manning and Dulk, 2001; Zarka et al., 2004), the flux density of galactic back ground radiation is $S=-190 \mathrm{dBW} / \mathrm{m}^{2} \mathrm{~Hz}$ at $5 \mathrm{MHz}$. If we consider that the effective length of the LRS dipole antenna is $L_{e}=\lambda / \pi=$ $19.1 \mathrm{~m}$, free space impedance is $Z_{0}=120 \pi \Omega$, and receiving bandwidth of LRS is $3 \mathrm{kHz}$, the electric field of galactic noise and the voltage produced at the LRS preamplifier input are obtained as follows:

$$
\begin{gathered}
E=\sqrt{S Z_{0} \Delta f}=0.336 \mu \mathrm{V} / \mathrm{m} \\
V=E L_{e}=6.42 \mu \mathrm{V}
\end{gathered}
$$

Therefore, echoes with electric field intensity larger than $-10 \mathrm{~dB} \mu \mathrm{V} / \mathrm{m}$, which produce a voltage larger than $16 \mathrm{~dB} \mu \mathrm{V}$ at the LRS preamplifier input, have to be detectable by the LRS. Based on Zarka (1998) and Zarka et al. (2004), the intensity of Jovian HOM and DAM emissions in active cases is estimated to be $-190 \mathrm{dBW} / \mathrm{m}^{2} \mathrm{~Hz}$ at the lunar orbit. In order to avoid the lunar subsurface echo and the planetary radio waves from being masked by artificial RE noises, limit for RE noises (Fig. 2) was determined. The RE limit for instruments outside the spacecraft panels is $-10 \mathrm{~dB} \mu \mathrm{V} / \mathrm{m}$ in a frequency range from $14 \mathrm{kHz}$ to $30 \mathrm{MHz}$, which is just equal to the galactic noise level at $5 \mathrm{MHz}$. As reported by the previous studies of galactic noise spectrum (Dulk et al., 2001; Manning and Dulk, 2001; Zarka et al., 2004), the galactic noise level in a frequency range below $1 \mathrm{MHz}$ is lower than that at $5 \mathrm{MHz}$. The RE limit below $1 \mathrm{MHz}$ was, however, determined to be larger than $-10 \mathrm{~dB} \mu \mathrm{V} / \mathrm{m}$ because the ambient noise level in the first EMC test in 2004 was as high as $-10 \mathrm{~dB} \mu \mathrm{V} / \mathrm{m}$. It was inferred that the external power supply of the spacecraft brought the external noises into the anechoic chamber in the first EMC test. The external noises were, however, suppressed by on-board battery operation in the final EMC test in 2005. As a result, spacecraft noises even below the RE limit were also evaluated precisely. As for the instru- 

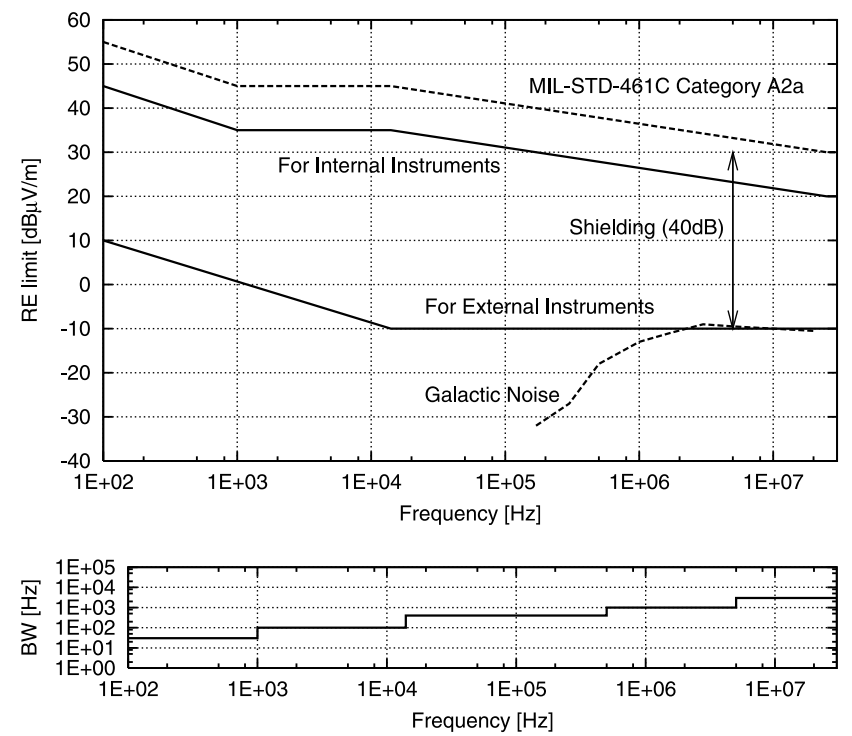

Fig. 2. RE limit for the SELENE spacecraft. Upper panel shows the allowed RE noise levels for instruments inside and outside the spacecraft panels as a function of frequency. Galactic noise level and RE limit of MIL-STD-461C Category A2a are indicated by dashed lines. Lower panel indicates bandwidth for RE measurements.

ments inside the spacecraft, another RE limit was determined. Based on shielding tests with dummy panels, it was confirmed that more than $40 \mathrm{~dB}$ of shielding effects can be expected by the spacecraft panels in a frequency range up to $30 \mathrm{MHz}$. Most of spacecraft BUS equipments are basically designed to meet the MIL-STD-461C Category A2a (US Department of Defence, 1999), general EMC standard for airborne and spaceborne instruments. However, as shown in Fig. 2, the MIL-STD limit at $5 \mathrm{MHz}$ is a little higher than $30 \mathrm{~dB} \mu \mathrm{V} / \mathrm{m}, 40 \mathrm{~dB}$ higher than the RE limit for instruments outside the spacecraft panels. RE limit for instruments inside the spacecraft panels was, therefore, determined to be just $10 \mathrm{~dB}$ stricter than the MIL-STD-461C Category A2a.

Not only the RE limit but also the CM-current limit are necessary for LRS observations. In order to avoid the potential differences between LRS preamplifier inputs from exceeding $16 \mathrm{~dB} \mu \mathrm{V}$, both (1) unbalanced currents in the interconnecting cables, which are also called "line conductive noise", or "LC noise" in the SELENE development team, and (2) impedance between the spacecraft panels, $Z_{I P}$, should be controlled. CM-current limit is shown in Fig. 3. The CE limit of MIL-STD-461C is defined only for each of the HOT and RTN lines of the power supply via which other instruments can be disturbed. In the case of the spacecraft EMC, however, not only the CM currents those in power supply lines but also those in signal lines can produce potential difference between spacecraft panels. The CM-current limit of SELENE EMC is, therefore, applied for all interconnecting cables of all instruments onboard the SELENE spacecraft. CM currents can be measured by clamping the current probe around both HOT and RTN lines of the cables. In the early phase of spacecraft development, $Z_{I P}$ was estimated as $0.01-0.03 \Omega$. This $Z_{I P}$ estimation was, however, not based on the measurements of actual spacecraft panels but based on measurements of

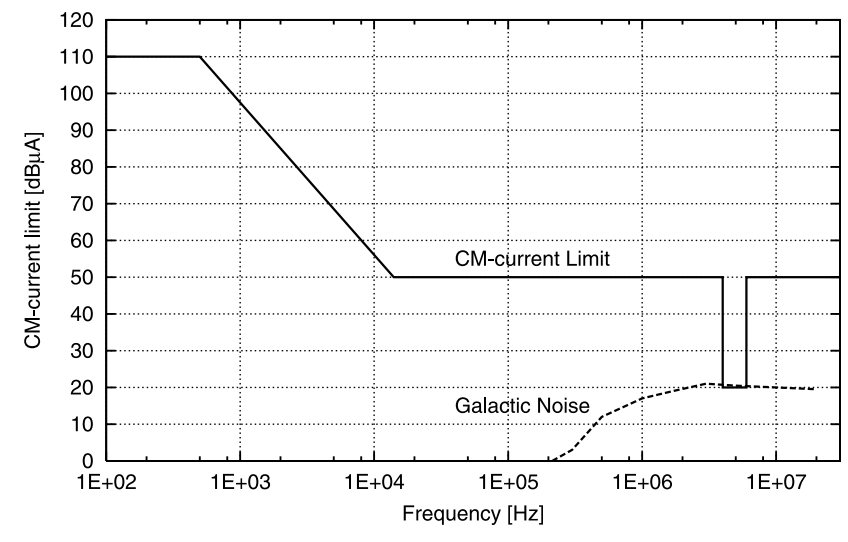

Fig. 3. CM-current limit for the SELENE spacecraft. Allowed CM-current level is indicated as a function of frequency. In particular, the regulation level in a frequency range from 4 to $6 \mathrm{MHz}$ is reduced to $20 \mathrm{~dB} \mu \mathrm{A}$. Equivalent level of galactic noise is indicated by a dashed line.

dummy panels and equivalent circuit analyses. Based on the estimated $Z_{I P}$, the CM-current limit was determined to be $50 \mathrm{~dB} \mu \mathrm{A}$. However, the first system EMC test revealed that $Z_{I P}$ is $0.3-1 \Omega$ based on measurements of the potential difference between actual spacecraft panels. It was difficult to change EMC limits because the development of onboard instruments was almost finished. Considering the importance of the lunar subsurface radar sounding in the SELENE mission, the CM-current limit in a frequency range from 4 to $6 \mathrm{MHz}$ especially was reduced to $20 \mathrm{~dB} \mu \mathrm{A}$.

The EMC performance of each instrument was checked by RE and CM-current measurements. RE noise level from the components was measured using a calibrated monopole antenna. It is generally difficult to set up the configuration in which ambient noise is enough low for RE measurement. The component test equipment, such as external power supply, controller, and data acquisition PCs, often become noise sources and mask RE noises from the instrument itself. CM currents in each cable between subcomponents were measured by clamp-on current probe, as shown by Fig. 4. Because the current probe measures net currents just inside the clump core, it is usually easy to set up the CM-current measurements configuration even in the ordinary working space. CM-current control was, therefore, useful and effective enough for noise reduction of each instrument in the factory.

The final system EMC test of the SELENE spacecraft was carried out from October 20 to 22,2005 . In the final system EMC test, the RE and CM-current noises from the whole SELENE spacecraft system were directly measured by the LRS receivers with dummy antenna whose element length is $5 \mathrm{~m}$, or $1 / 3$ of fully extended antenna. The configuration of the dummy antenna is shown in Fig. 5. Furthermore, the potential difference $V_{I P}$ and impedance $Z_{I P}$ between the spacecraft panels were also measured to evaluate CM-current noises. $V_{I P}$ was measured using a differential amplifier with a gain of $20 \mathrm{~dB}$ and a spectrum analyzer. The potential difference produced between the spacecraft panels can be determined by the $V_{I P}$ measurement. Final evaluation of the SELENE EMC performance was carried out based mainly on LRS data. It should be especially noted 


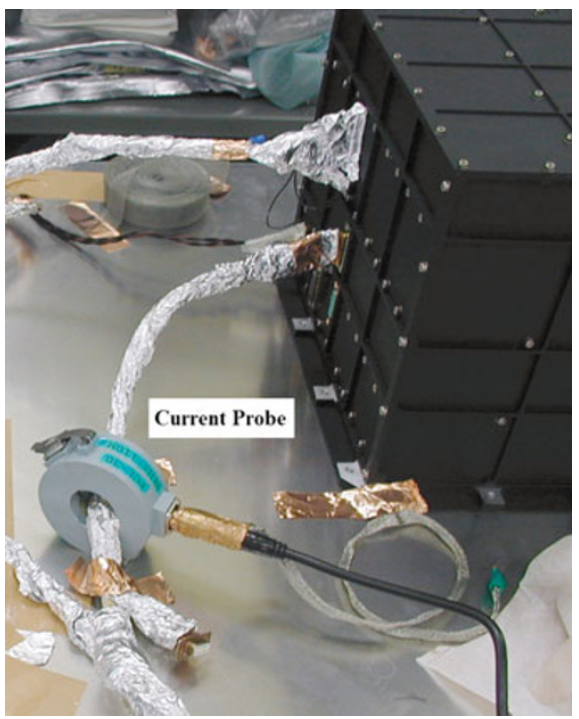

Fig. 4. CM-current measurement in the component EMC test. A clamp-on current probe is utilized for the measurements of CM currents in the cables connecting multiple components.

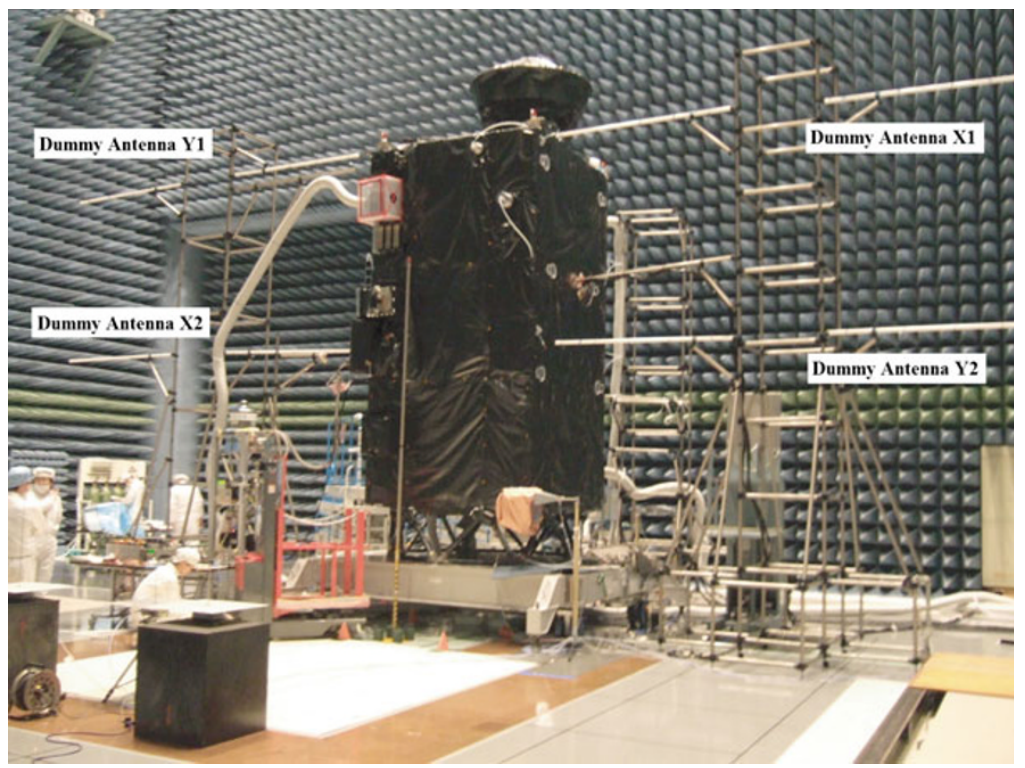

Fig. 5. Configuration of the dummy antenna elements in the final EMC test from October 20 to 22, 2005.

that the spacecraft was disconnected from the external test equipment during EMC measurements: the external power supply lines are removed from the spacecraft and the spacecraft was operated with the onboard battery. By this effort, the ambient noises were drastically reduced, which enabled us to accurately evaluate SELENE EMC performance.

\section{Results of the Final System EMC Test}

RE and CM-current noises of the SELENE spacecraft measured by the sweep frequency analyzer (NPW-DS, $<30 \mathrm{MHz}$ ) and wide-band receiver (NPW-W, $<12.5 \mathrm{MHz}$ ) of the LRS are indicated in Figs. 6 and 7, respectively. The green curves indicate the minimum detectable level of the LRS receivers measured by GND-CAL operation which connect signal input line to the preamplifier ground. Minimum detectable sensitivity is also related to the bandwidth of the receivers. Bandwidth of NPW-DS is $3 \mathrm{kHz}$ in a fre- quency range below $6.7 \mathrm{MHz}$ and $10 \mathrm{kHz}$ in a frequency range above $6.7 \mathrm{MHz}$. Bandwidth of NPW-W is $6 \mathrm{kHz}$ in all frequency ranges. A small enhancement around 1$4 \mathrm{MHz}$ is also seen in the minimum detectable level of the NPW-W receiver; This is probably due to some frequency characteristics of NPW-W receiver. The blue curves indicate noise spectrum measured by the LRS receivers when all BUS and scientific instruments are being operated. The spectrum peak around $12 \mathrm{MHz}$ is antenna resonance of the dummy antenna. Antenna resonance results from the inductance and capacitance of the antenna and input capacitance of the preamplifier. Therefore, the occurrence of antenna resonance can not be avoided. It is, however, possible to moderate frequency dependence of receiving gain by adding resistance in series with the antenna. Based on an analysis of the equivalent circuit of antenna and preamplifier, it is inferred that antenna resonance occurs around 


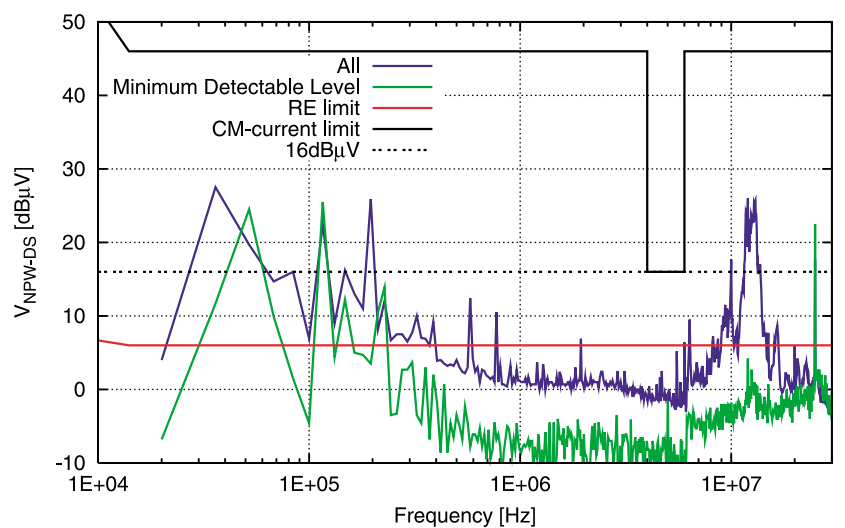

Fig. 6. Noise spectrum measured by the LRS/NPW-DS receiver, which covers a frequency range from $20 \mathrm{kHz}$ to $30 \mathrm{MHz}$ with a sweep frequency analyzer (blue curve). The green curve indicates the minimum detectable level of the LRS/NPW-DS receiver. The CM-current limit is indicated by black line. Ideal CM-current limit, $16 \mathrm{~dB} \mu \mathrm{V}$, is shown by a dashed line. RE limit, $-10 \mathrm{~dB} \mu \mathrm{V} / \mathrm{m}$ or $6 \mathrm{~dB} \mu \mathrm{V}$ at LRS preamplifier input, is indicated by a red line.

6.5 and 17.6 $\mathrm{MHz}$ with a fully extended flight antenna. Due to the effect by the series resistance mentioned above, deviations of receiving gain around the antenna resonance have been suppressed within $10-15 \mathrm{~dB}$. The antenna resonance does not change the $\mathrm{S} / \mathrm{N}$ ratio but decreases the dynamic range of the receiver. The dynamic range of the LRS sounder receiver is about $70 \mathrm{~dB}$ (Ono et al., 2008). It is inferred that the enhancement of receiving gain with 10$15 \mathrm{~dB}$ does not cause serious problems on the observations. The CM-current limit is indicated by a black line. Assuming that impedance between the spacecraft panels is $0.6 \Omega$, the CM-current limit at the LRS preamplifier input is estimated to be $16 \mathrm{~dB} \mu \mathrm{V}$. The CM-current limit is, however, too loose in a frequency range outside 4-6 MHz due to practical reasons associated with the spacecrafts development. The reference level of $16 \mathrm{~dB} \mu \mathrm{V}$ is also indicated as the ideal CM-current limit. The RE limit, which is just equal to galactic noise level, is indicated by a red line. RE noise at a level equal to that of the galactic noise produces voltage of $16 \mathrm{~dB} \mu \mathrm{V}$ at the LRS preamplifier input when it is detected by the fully extended antenna with a tip-to-tip length of $30 \mathrm{~m}$. However, by using a dummy antenna for the EMC test, with a length $1 / 3$ that of the fully extended antenna, the detected level of the RE noise is reduced to 6 $\mathrm{dB} \mu \mathrm{V}$. It should be noted that RE noise level measured in the final EMC test becomes about $10 \mathrm{~dB}$ smaller than that in the flight configuration with a fully extended antenna. Except for frequency range around $12 \mathrm{MHz}$, or antenna resonance, there is no broadband noise whose level exceeds the CM-current limit in a frequency range from 0.3 to 30 $\mathrm{MHz}$. As for a frequency range below $0.3 \mathrm{MHz}$, minimum detectable levels of the NPW-DS and NPW-W receivers are unfortunately larger than the CM-current limit. The NPW receivers are not designed for low frequency ranges. In order to cover the frequency range below $1 \mathrm{MHz}$, waveform capture (WFC) receivers of the LRS are operated with a radar sounder and NPW receivers (Kasahara et al., 2008).

Radar sounding of the lunar subsurface structure is performed using a frequency-modulated continuous wave

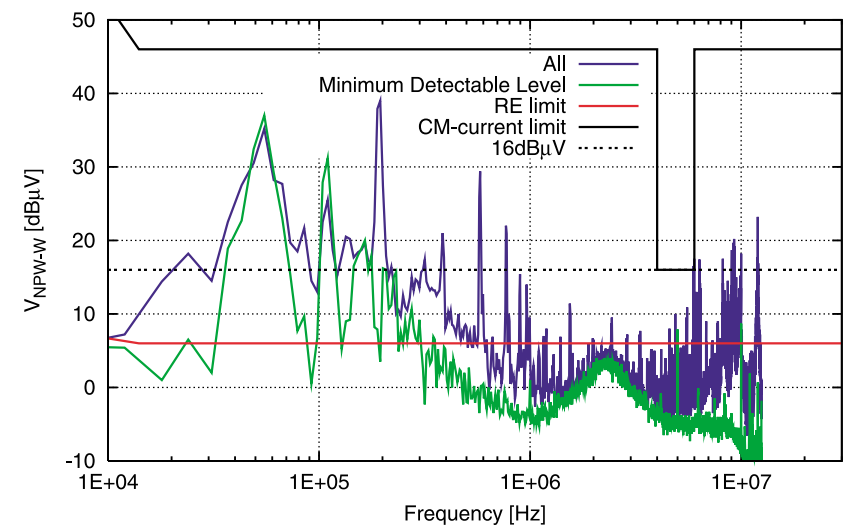

Fig. 7. Noise spectrum measured by the LRS/NPW-W receiver, which covers a frequency range up to $12.5 \mathrm{MHz}$ with wide-band receiver (blue curve). The green curve indicates the minimum detectable level of the LRS/NPW-W receiver. CM-current limit is indicated by a black line. Ideal CM-current limit, $16 \mathrm{~dB} \mu \mathrm{V}$, is shown by a dashed line. RE limit, $-10 \mathrm{~dB} \mu \mathrm{V} / \mathrm{m}$ or $6 \mathrm{~dB} \mu \mathrm{V}$ at LRS preamplifier input, is indicated by a red line.

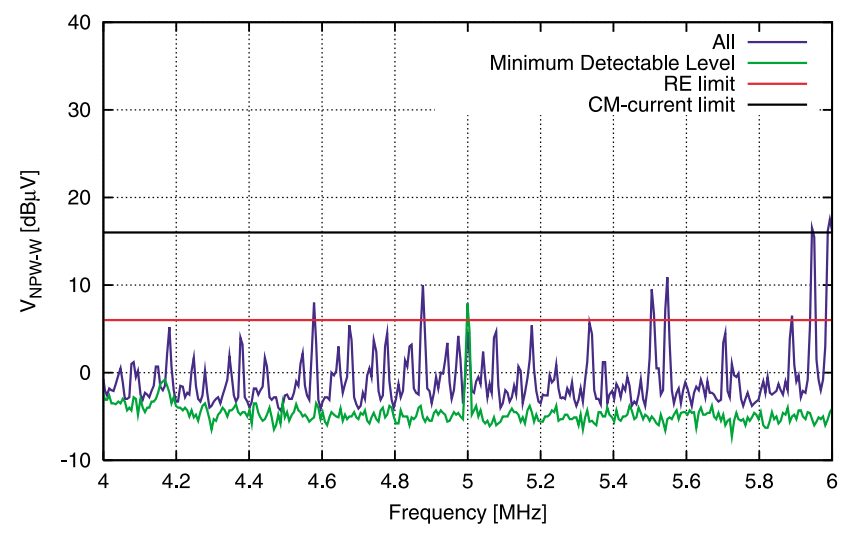

Fig. 8. Noise spectrum in a frequency range from 4 to $6 \mathrm{MHz}$ measured by the LRS/NPW-W receiver (blue curve). The green curve indicates the minimum detectable level of the LRS/NPW-W receiver. CM-current limit is indicated by black line. RE limit, $-10 \mathrm{~dB} \mu \mathrm{V} / \mathrm{m}$ or $6 \mathrm{~dB} \mu \mathrm{V}$ at LRS preamplifier input, is indicated by a red line.

(FMCW) method in a frequency range from 4 to $6 \mathrm{MHz}$. EMC performance in this frequency range is essential for the lunar subsurface radar sounding. In the LRS receiver, the echo signal of the transmitted pulse whose frequency is swept from 4 to $6 \mathrm{MHz}$ is mixed with a local signal whose frequency is also swept from 4 to $6 \mathrm{MHz}$ with a given delay time. At the intermediate frequency (IF) output, the echo signal is converted to an intermediate signal with a fixed frequency while the narrowband noises with fixed frequency $f$ are converted to an intermediate signal with a swept frequency from $f-4 \mathrm{MHz}$ to $f-6 \mathrm{MHz}$. The input power of narrowband noises within a frequency range from 4 to $6 \mathrm{MHz}$ is, therefore, widely spread in a frequency range from 0 to $2 \mathrm{MHz}$ at the IF output. Increase of the IF output level by single narrowband noise may be small and limited. However, it should be confirmed that the total increase of the IF output level by all noises within 4-6 MHz does not exceed EMC limits. The IF output level can be evaluated by averaging noise power within a frequency range from 4 to $6 \mathrm{MHz}$. The noise power averaged within $4-6 \mathrm{MHz}$ is 


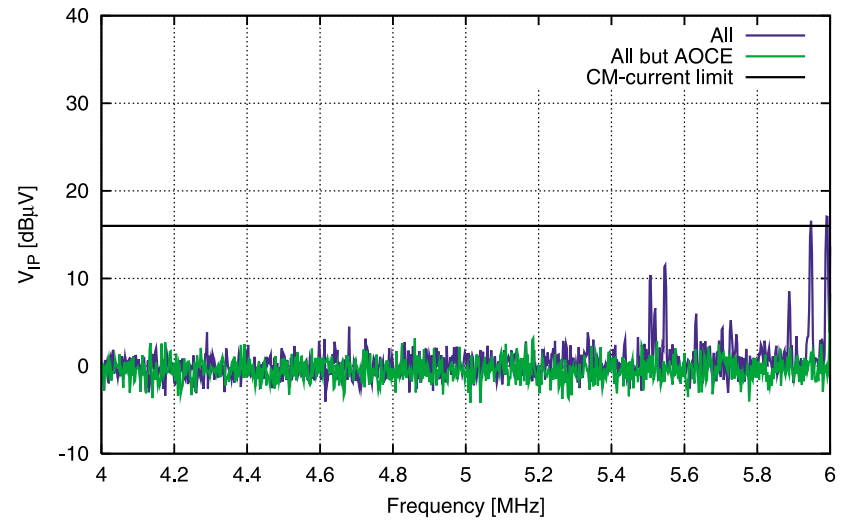

Fig. 9. Potential difference $V_{I P}$ between the $+\mathrm{Y}$ panel and $+\mathrm{Z}$ panel. The blue curve indicates $V_{I P}$ measured when all BUS and scientific instruments were in operation. The green curve indicates $V_{I P}$ measured when all instruments but AOCE were in operation. $V_{I P}$ at 5.50, 5.55, 5.95 , and $5.99 \mathrm{MHz}$ are quite equal to the narrowband noise levels detected by the LRS.

given as follows:

$$
P_{I F}=\frac{3 \mathrm{kHz}}{2 \mathrm{MHz}} \int_{4 \mathrm{MHz}}^{6 \mathrm{MHz}} P_{R F}(f) d f,
$$

where $P_{R F}(f)$ is the spectral density of noise power fed into the LRS receiver input as a function of radio frequency $f$, $P_{I F}$ is noise power at the IF output, which is received by the LRS sounder receiver with a bandwidth of $3 \mathrm{kHz}$. RE and CM-current noises in a frequency range from 4 to $6 \mathrm{MHz}$ are indicated in Fig. 8. The RE limit, $6 \mathrm{~dB} \mu \mathrm{V}$, is indicated by a red line. There is no broadband noise with a larger level than the RE limit. There are, however, several narrowband noises whose level is larger than the RE limit. Based on the noise spectrum shown in Fig. 8, the noise level averaged within $4-6 \mathrm{MHz}$ is estimated as $1.9 \mathrm{~dB} \mu \mathrm{V}$, which is $14 \mathrm{~dB}$ lower than the CM-current limit and $4 \mathrm{~dB}$ lower than the RE limit.

It has been confirmed by the component EMC tests that the attitude and orbit control electronics (AOCE) unit causes the CM-current noises at 5.50, 5.55, 5.95, and $5.99 \mathrm{MHz}$ and that the remote terminal unit (RTU) causes the CM-current noises at $5.99 \mathrm{MHz}$. For the purpose of identification of CM-current noises in the LRS data, potential differences between the spacecraft panels, $V_{I P}$, were measured. $V_{I P}$ between the $+\mathrm{Y}$ and $+\mathrm{Z}$ panels is shown in Fig. 9. There are intense narrowband noises at 5.50, 5.55, 5.95 , and $5.99 \mathrm{MHz}$ in the $V_{I P}$ spectrum obtained when all BUS and scientific instruments were in operation, as indicated by the blue curve. The noise levels of the narrowband noises are quite equal to the noise levels detected by the NPW-W receiver, shown in Fig. 8. The noises at 5.50, 5.55 , and $5.95 \mathrm{MHz}$, however, disappear in the $V_{I P}$ spectrum obtained when all instruments but AOCE were in operation. It is therefore confirmed that narrowband noises at 5.50, 5.55, 5.95, and 5.99 MHz measured by the LRS are CM-current noises, probably from AOCE and RTU. For NPW-W receiver measurements, Y1 and Y2 antennas were utilized. Because all preamplifiers are installed on the $+\mathrm{Z}$ panel, GND levels of the preamplifiers are considered to be almost the same. The AC voltage has to be induced at

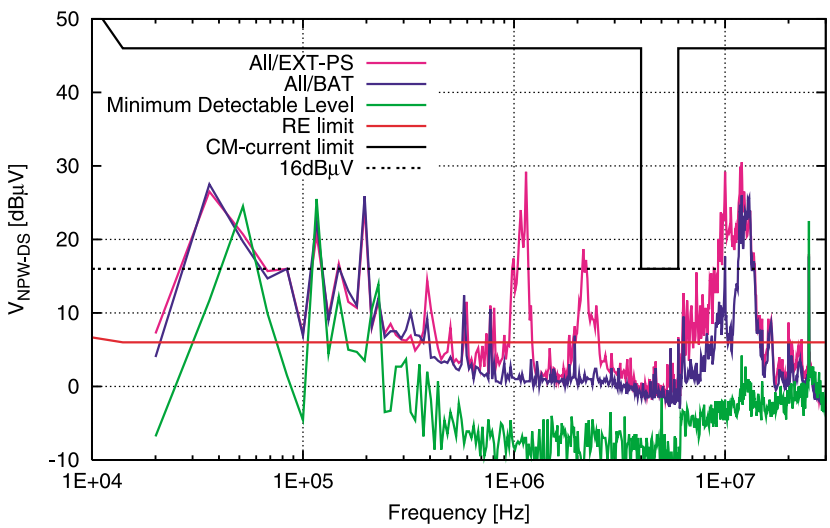

Fig. 10. Comparison of noise levels during the external power supply operation (pink line) and during the onboard battery operation (blue line) in a frequency range up to $30 \mathrm{MHz}$. EMC limits are also indicated, as in Fig. 6.

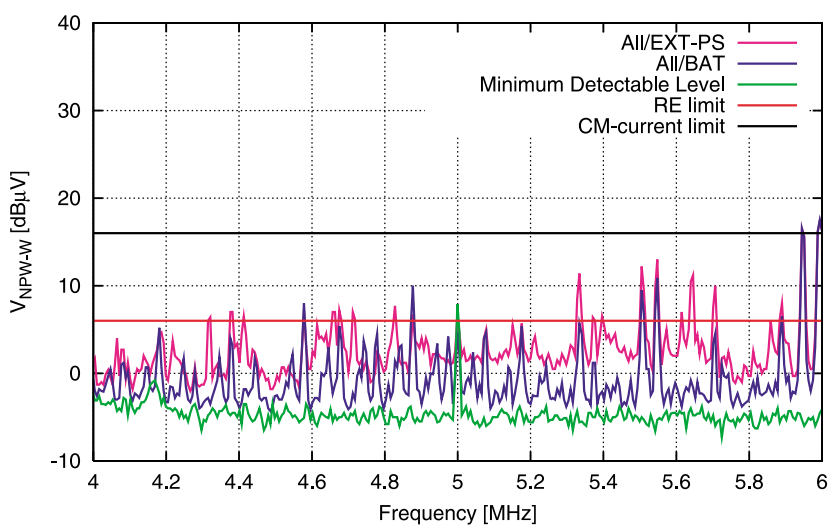

Fig. 11. Comparison of noise levels during the external power supply operation (pink line) and during the onboard battery operation (blue line) in a frequency range from 4 to $6 \mathrm{MHz}$. EMC limits are also indicated, as in Fig. 8.

the preamplifier signal input. The AC voltage was probably generated around the $+\mathrm{Y}$ and $+\mathrm{Z}$ panels whose potential was oscillated by the CM-current noises. It is inferred that the $\mathrm{AC}$ voltage generated around $+\mathrm{Y}$ panel was induced at the preamplifier input for the Y1 antenna, which is located near the $+\mathrm{Y}$ panel. In the component EMC test, the measured levels of CM-current noises at 5.50, 5.55, 5.95, and $5.99 \mathrm{MHz}$ are $23,24,25$, and $29 \mathrm{~dB} \mu \mathrm{A}$, respectively. Based on the current level, the inter-panel impedance $Z_{I P}$ is estimated as $0.2 \Omega$. $Z_{I P}$ was also measured by the differential amplifier with impressing AC voltage between the spacecraft panels by signal generator before the spacecraft operations were started up. Measured $Z_{I P}$ between $+\mathrm{Y}$ and $+\mathrm{Z}$ panels was, however, $5 \Omega$.

The comparison of noise levels during external power supply (EXT-PS) operation and onboard battery (BAT) operation is shown in Fig. 10. Intense noises are found around $1 \mathrm{MHz}, 2 \mathrm{MHz}$, and from 6 to $12 \mathrm{MHz}$ during EXT-PS operation. The intensity of EXT-PS noises is up to $30 \mathrm{~dB} \mu \mathrm{V}$, which is $15 \mathrm{~dB}$ larger than the CM-current limit. Figure 11 shows the comparison of noise level in a frequency range from 4 to $6 \mathrm{MHz}$. With onboard battery operation, the broadband noise level was reduced to just $1-2 \mathrm{~dB}$ above 
the minimum detectable level of the LRS receiver, which was a much lower level than expected.

\section{Discussion and Conclusions}

The EMC control procedures of the SELENE spacecraft for the LRS observations were performed based on the following EMC standard:

(1) Galactic noise level, $-190 \mathrm{dBW} / \mathrm{m}^{2} \mathrm{~Hz}$ at $5 \mathrm{MHz}$, was used as the critical level for detection of the lunar subsurface echoes from a depth of $5 \mathrm{~km}$.

(2) Considering the critical level and ambient noise level measured in the first system EMC test in 2004, RE limit for instruments outside the spacecraft panels was determined to be $-10 \mathrm{~dB} \mu \mathrm{V} / \mathrm{m}$. The RE limit for instruments inside the spacecraft panels was determined to be at a $10 \mathrm{~dB}$ lower level than MIL-STD-461 Category A2a because a $40 \mathrm{~dB}$ of shielding effect of the spacecraft panels can be expected.

(3) Considering the critical level and estimated impedance between the spacecraft panels, CM-current limit was determined to be $50 \mathrm{~dB} \mu \mathrm{A}$ at first. After the first system EMC test, CM-current limit in a frequency range from 4 to $6 \mathrm{MHz}$ was reduced to $20 \mathrm{~dB} \mu \mathrm{A}$ because inter-panel impedance was larger than the estimated one.

The SELENE EMC limits have been modified not only based on scientific objectives but also based on the practical reasons. In order to ensure the planetary radio wave observation, the CM-current limit in all frequency ranges up to $30 \mathrm{MHz}$ should be changed to $20 \mathrm{~dB} \mu \mathrm{A}$. Considering that the development of each instrument was almost finished at the first system EMC test, the CM-current limit was changed only in the frequency range from 4 to $6 \mathrm{MHz}$, which is the most important frequency range of the LRS mission.

Based on the results of the final system EMC test in 2005, the EMC performance of the SELENE spacecraft for the LRS observations is summarized as follows:

(1) There is no broadband noise at a level higher than the RE limit in a frequency range from 4 to $6 \mathrm{MHz}$. There are, however, some narrowband noises whose level exceeds even the CM-current limit in the frequency range. Based on the noise spectrum, the noise level averaged within 4$6 \mathrm{MHz}$ is estimated as $1.9 \mathrm{~dB} \mu \mathrm{V}$ at the LRS preamplifier input, or $14 \mathrm{~dB}$ below the CM-current limit.

(2) In a frequency range from 0.3 to $30 \mathrm{MHz}$, there is no broadband noise at a level higher than the CM-current limit. There are, however, many narrowband noises whose level exceeds the CM-current limit.

(3) In a frequency range from 0.02 to $0.3 \mathrm{MHz}$, the minimum sensitivity threshold of the LRS receiver is unfortunately higher than the CM-current limit.

(4) The potential difference between spacecraft panels, $V_{I P}$, shows a noise spectrum that is quite similar to that observed by the LRS. Narrowband noises at 5.50, 5.55, 5.95, and $5.99 \mathrm{MHz}$ were confirmed to be CM-current noises from AOCE and RTU. Based on the $V_{I P}$ and AOCE CM current measured in the component EMC test, the impedance between spacecraft panels, $Z_{I P}$, was estimated to be $0.2 \Omega$. However, $Z_{I P}$ directly measured by differential amplifier and signal generator was $5 \Omega$.

(5) In the SELENE final system EMC test, the spacecraft was operated with the onboard battery when EMC measurements were performed. The intense noises with a level up to $30 \mathrm{~dB} \mu \mathrm{V}$ during external power supply operation drastically disappear during onboard battery operation.

It can be concluded that the noise level of the SELENE spacecraft is low enough to perform the lunar subsurface radar sounding by the LRS. The estimated FMCW sounder noise level, $1.9 \mathrm{~dB} \mu \mathrm{V}$, was $14 \mathrm{~dB}$ lower than the CMcurrent limit and $4 \mathrm{~dB}$ lower than the RE limit. Most of the noise power is probably due to the intense narrowband noises at 5.50, 5.55, 5.95, and 5.99 MHz. Based on the measurements of the potential difference between the spacecraft panels, these noises are not RE noises but CM-current noises. Therefore, because the FMCW sounder noise level is less than the CM-current limit, the noises will not cause any EMC problems in the radar sounding observations performed by the LRS.

Probably due to strict CM-current limit, the noises within a frequency range from 4 to $6 \mathrm{MHz}$ are generally well suppressed in the low level. There are, however, many narrowband noises whose level is larger than CM-current limit in frequency ranges from $20 \mathrm{kHz}$ to $4 \mathrm{MHz}$ and from $6 \mathrm{MHz}$ to $30 \mathrm{MHz}$. These are, off course, unfavorable for the observations of radio waves propagating from the Jupiter and plasma waves generated around the moon. However, they will not cause any fatal difficulty with the observations if the number of noises is limited and the LRS preamplifiers are not saturated by the noises.

In the SELENE final system EMC test, two EMC measurement methods which have never done in the previous satellite EMC test were carried out: (1) measurements of the potential difference between the spacecraft panels, $V_{I P}$, and (2) onboard battery operation during EMC measurements.

In the noise spectrum measured by LRS, it is difficult to distinguish RE noises detected by the dummy antenna and CM-current noises impressed by the spacecraft panels. The $V_{I P}$ measurement clearly shows whether the narrowband noise is CM-current noise or not. Some narrowband noises detected by the LRS were successfully identified as CM-current noises based on $V_{I P}$ measurements. The potential difference impressed on the LRS preamplifier inputs depends not only on CM-current level but also on the impedance between spacecraft panels, $Z_{I P}$. $V_{I P}$ measurements are also important for evaluating the $Z_{I P}$ control results. As for CM-current noises from AOCE, the $Z_{I P}$ estimated from $V_{I P}$ and the CM-current level was quite different from directly measured $Z_{I P}$. It is unlikely that CMcurrent level of AOCE was drastically reduced in configuration given that AOCE is installed on the spacecraft panel with lower impedance than in the component EMC test. It should also be noted that directly measured $Z_{I P}$ can be affected by the inductance of the cables used for connection between the spacecraft panels and the measurement equipments.

Onboard battery operation has not been usually used in the system EMC tests of the previous satellites because (1) the onboard battery operation was usually limited in the spacecraft ground test to avoid degradation of the onboard battery and (2) ambient noise reduction of the EMC test site was believed to be difficult. In the SELENE case, we 
fortunately had the opportunity to carry out EMC measurements in onboard battery operation by using the redundant battery to be replaced. The comparison between the noise spectra measured during external power supply operation and onboard battery operation clearly shows the advantage of EMC measurements during onboard battery operation. It was believed that weak RE noises as large as the LRS minimum detectable level can not be measured in the anechoic chamber of TKSC. However, as shown in Fig. 11, $\mathrm{RE}$ noises with a level of $-20 \mathrm{~dB} \mu \mathrm{V} / \mathrm{m}$ can be detected by using onboard battery operation.

As a result of the strict EMC control for all instruments and the whole system of the SELENE spacecraft, it was finally confirmed that the EMC performance of the SELENE spacecraft meets the requirements for the lunar subsurface radar sounding by the LRS. Based on the SELENE case, recommendations for EMC control and evaluation in future satellite missions are presented as follows:

(1) CM-current control is useful and effective enough in the development phase of each instrument. An anechoic chamber is not necessary for CM-current measurements. All you need to do is just clamp the current probe on the cable between the components. The easiness of EMC measurements will increase the opportunity to improve the EMC performance of the instruments. In the SELENE case, noise level in a frequency range from 4 to $6 \mathrm{MHz}$ is clearly lower than that in the other frequency range. CM-current control is enough effective for reduction of instrument noises.

(2) $V_{I P}$ measurements are useful to distinguish RE and CMcurrent noises detected by the onboard wave receiver. In order to reduce $V_{I P}$, it is also important to control $Z_{I P}$. In the SELENE case, determination of $Z_{I P}$ is controversial, and the method for direct measurements of $Z_{I P}$ should be improved. It is at least sure that directly measured $V_{I P}$ coincides with CM-current level measured by the onboard wave receiver without any assumption of $Z_{I P}$ and CM-current levels, as clearly confirmed in the SELENE case.

(3) Onboard battery operation is extremely effective for ambient broadband noise reduction. It was believed that EMC performance of the satellite can not be fully evaluated in the ground test due to large ambient noise even in the anechoic chamber. In the SELENE case, ambient broadband noise level can be reduced to just $1-2 \mathrm{~dB}$ above the minimum detectable level of the onboard wave receiver by utilizing onboard battery operation for EMC measurements. It is highly recommended that battery operation be effectively utilized for the EMC tests.

The EMC control and evaluation methods improved through the SELENE spacecraft development, as mentioned above, will also be useful for future satellite missions which are to perform radar soundings and natural wave observations with high sensitivity.

Acknowledgments. The SELENE mission has been organized by the Japan Aerospace Exploration Agency (JAXA). The authors would like to express their thanks to the all members of SELENE development team. The authors are also grateful to members of SELENE EMC working group.

\section{References}

Dulk, G. A., W. C. Erickson, R. Manning, and J.-L. Bougeret, Calibration of low-frequency radio telescopes using the galactic background radiation, Astron. Astrophys., 365, 294-300, 2001.

Kasahara, Y., Y. Goto, K. Hashimoto, T. Imachi, A. Kumamoto, T. Ono, and H. Matsumoto, Plasma wave observation using waveform capture in the Lunar Radar Sounder on board the SELENE spacecraft, Earth Planets Space, 60, this issue, 341-351, 2008.

Kimura, I., K. Hashimoto, I. Nagano, T. Okada, M. Yamamoto, T. Yoshino, H. Matsumoto, M. Ejiri, and K. Hayashi, VLF observations by the Akebono (EXOS-D) satellite, J. Geomag. Geoelectr., 42, 459-478, 1990.

Kobayashi, T. and T. Ono, Estimation of planetary surface roughness using radio sounder A-scope data, J. Geophys. Res., 111, E06S10, doi:10.1029/2005JE002575, 2006.

Kobayashi, T. and T. Ono, SAR/InSAR observation by an HF sounder, $J$. Geophys. Res., 112, E03S90, doi:10.1029/2005JE002576, 2007.

Kobayashi, T., H. Oya, and T. Ono, A-scope analysis of subsurface radar sounding of lunar mare region, Earth Planets Space, 54, 973-982, 2002a.

Kobayashi, T., H. Oya, and T. Ono, B-scan analysis of subsurface radar sounding of lunar highland region, Earth Planets Space, 54, 983-991, 2002b.

Manning, R. and G. A. Dulk, The Galactic background radiation from 0.2 to $13.8 \mathrm{MHz}$, Astron. Astrophys., 372, 663-666, 2001.

Matsumoto, H., I. Nagano, R. R. Anderson, H. Kojima, K. Hashimoto, M. Tsutsui, T. Okada, I. Kimura, Y. Omura, and M. Okada, Plasma wave observations with Geotail spacecraft, J. Geomag. Geoelectr., 46, 59-95, 1994

Matsumoto, H., T. Okada, K. Hashimoto, I. Nagano, S. Yagitani, M. Tsutsui, Y. Kasaba, K. Tsuruda, H. Hayakawa, A. Matsuoka, S. Watanabe, H. Ueda, I. Kimura, Y. Kasahara, Y. Omura, T. Matsumura, T. Imachi, K. Ishisaka, and Y. Tateno, Low Frequency plasma wave Analyzer (LFA) onboard the PLANET-B spacecraft, Earth Planets Space, 50, 223-228, 1998

Ono, T. and H. Oya, Lunar Radar Sounder (LRS) experiment onboard the SELENE spacecraft, Earth Planets Space, 52, 629-637, 2000.

Ono, T, H. Oya, A. Morioka, A. Kumamoto, K. Kobayashi, T. Obara, and T. Nakagawa, Plasma Waves and Sounder (PWS) experiment onboard the Planet-B Mars orbiter, Earth Planets Space, 50, 213-221, 1998.

Ono, T., A. Kumamoto, Y. Yamaguchi, A. Yamaji, T. Kobayashi, Y. Kasahara, and H. Oya, Instrumentation and observation target of the Lunar Radar Sounder (LRS) experiment on-board the SELENE spacecraft, Earth Planets Space, 60, this issue, 321-332, 2008.

Oya, H, A. Morioka, K. Kobayashi, M. Iizima, T. Ono, H. Miyaoka, T. Okada, and T. Obara, Plasma wave observation and sounder experiments (PWS) using the Akebono (EXOS-D) satellite-Instrumentation and initial results including discovery of the high altitude equatorial plasma turbulence, J. Geomag. Geoelectr., 42, 411-442, 1990.

Tsutsui, M., H. Kojima, I. Nagano, H. Sato, T. Okada, H. Matsumoto, T. Mukai, and M. Kawaguchi, Magnetic radiations from harness wires of spacecraft, IEICE Trans. Commun., E75-B, 174-182, 1992.

US Department of Defense, Characteristics of subsystems and equipment MIL-STD-461E, 1999.

Zarka, P., Auroral radio emissions at the outer planets: Observations and theories, J. Geophys. Res., 103(E9), 20,159-20,194, 1998.

Zarka, P., B. Cecconi, and W. S. Kurth, Jupiter's low-frequency radio spectrum from Cassini/Radio and Plasma Wave Science (RPWS) absolute flux density measurements, J. Geophys. Res., 109, A09S15, doi:10.1029/2003JA010260, 2004

A. Kumamoto (e-mail: kumamoto@stpp1.geophys.tohoku.ac.jp), T. Ono, Y. Kasahara, Y. Goto, Y. Iijima, and S. Nakazawa 